I.

Aus der chirurgischen Abteilnng des städt. Krankenhanses zu Altona.

\title{
Über Prothesen bei Exartikulation und Resektion des Unterkiefers.
}

\author{
Yon \\ Prof. Dr. Fritz König. \\ (Iit 6 Abbildungen.)
}

Eine an sich schon interessante Beobachtung einer enostalen Geschwulst des Unterkiefers, welche zur Exartikulation führte, bot mir Gelegenheit, der Frage näher zu treten, in welcher Weise gewöhnlich die Nachbehandlung dieser Operationen gehandhabt wird. Im ganzen sind das seltene Eingriffe und dementsprechend finden sich größere Mitteilungen darüber spärlich, anch scheint den paar beachtenswerten Publikationen zu diesem Gegenstand noch nicht volle Aufmerksamkeit geschenkt worden zu sein. Daher habe ich mich durch eine Umfrage bei einer ganzen Anzahl von Fachgenossen, welchen ich für die Freundlichkeit, mit der sie meine Fragen beantworteten, ansdrücklich danke, zu informieren gesucht. Aus den erhaltenen Mitteilungen geht hervor, daß eine Gleichmäßigkeit des Vorgehens auch nicht im entferntesten erreicht ist.

Die Entfernung eines Stückes ans dem Unterkiefer zieht immer eine Verschiebung der beiden Zahnreihen nach sich, und eine Veränderung in der Funktion der am Unterkiefer inserierenden Muskeln. Wenn der Kinnteil und eventuell benachbarte Stücke fortfallen, dann werden beide Kieferreste nach der Mittellinie gezogen, sie stellen sich im spitzen Winkel zueinander. Schon das ist störend, weil das Kanen beeinträchtigt wird; verhängnisvoller aber ist, daß durch Ablösung der betreffenden Muskelansätze das Zungenbein und die Zunge nach hinten sinken mit allen dadurch bedingten schweren Folgen. In etwas anderer Weise macht sich die einseitige 
Entfernung des Unterkiefers bemerkbar, durch welche die hier am Unterkiefer inserierenden Muskeln, vor allem der Pterygoid. int., ihren Halt verlieren. Der völlig aus dem Gleichgewicht geratene Unterkieferrest folgt willenlos dem Zug der an ihm inserierenden Muskeln, welche ihn vor allem auf die operierte Seite herüberzerren. So verläßt die Zahnreihe die korrespondierende des Oberkiefers, und zu der starken Entstellung tritt die Unmöglichkeit zu kauen, und wegen des fehlenden Halts überhaupt richtig zu beißen.

Den kurz skizzierten Folgeil der Operation zu begegnen, erweist sich zunächst am notwendigsten bei den durch Zurücksinken der Zunge lebensgefährlichen Resektionen des Kinnstücks. Hier kann die Zunge gleichzeitig fixiert werden an dem Ersatzstück. Bei allen Kontinuitätsresektionen wird eine entsprechend große Schiene, z. B. nach Partsch eine Metallschiene, in die Lücke gesetzt und mit den Resektionsenden durch Drahtnaht verbunden. Ist genügend Schleimhaut, um sie über der Schiene zusammenzunähen, vorhanden, so kann man versuchen, sie zu dauernder Einheilung zu bringen. In anderen Fällen muß oder kann an ihre Stelle später ein zahnärztlicher Ersatzteil, an den benachbarten Zähnen bzw. Alveolarrand befestigt, angebracht werden. Gelegentlich nur kann man davon aus besonderen Gründen einmal Abstand nehmen, doch ist der Erfolg dann immer ein beschränkter.

Bei dem 70 jăhrigen Patienten, dessen Abbildungen folgen (Fig. 1 u. 2), habe ich im Herbst 1902 ein groBes Carcinom von Zunge und Mundboden mit Resektion vom Kinnteil des Unterkiefers durch Medianschnitt exstirpiert, unmittelbar waren beide Kieferhälften durch eine von Herrn Zahnarzt Roloff angefertigte Metallplatte mit gabelförmigen Ansätzen - nach Hahl - verbunden worden, welche gleichzeitig der Zunge den notwendigen Halt gab. Der Patient machte eine gute Rekonvaleszenz durch, aber von einer definitiven Prothese, welche auch wegen Zahnmangels schwer anzubringen war, wurde abgesehen besonders mit Rücksicht auf das zu erwartende Rezidiv. In der Folge traten nun nach Entfernung der Metallplatte die beiden Kieferstümpe unter spitzem Winkel in der Mittellinie zusammen, so daß die Alveolarränder des Unterkiefers nach innen vou denen des Oberkiefers standen, aber die Bewegung war eine leidlich gute zum Kauen weicher Speisen, und die Entstellung, wie aus den Bildern hervorgeht, kaum vorhanden.

Weniger einfach als die Nachbehandlung der Kontinuitätsresektionen ist die Frage nach einem allen Anforderungen genügenden Ersatz gelöst nach Wegnahme einer ganzen Kieferhälfte bzw. der Exartikulation mit Fortnahme des den Ansatz der Muskeln bietenden Teiles der Pars horizontalis mandibulae. Diese Frage wird 
deshalb auch sehr verschieden beantwortet, und ihr näher zu treten, verlohnt sich der Mühe. Als Ziel unserer Bestrebungen gilt uns, nach der Exartikulation jede Entstellung durch Verschiebung der Alveolarreihe, sowie durch Einsinken der Gesichtshälfte zu verhüten und völlig feste Schluß-bzw. Kaufähigkeit der Kiefer zu erreichen.

Die Mittel, um die Verschiebung der Unterkieferstücke zu beheben, und sie in richtiger Stellung zu erhalten, hat man vielfach erst angewendet, nachdem die Wunde im wesentlichen verheilt war.

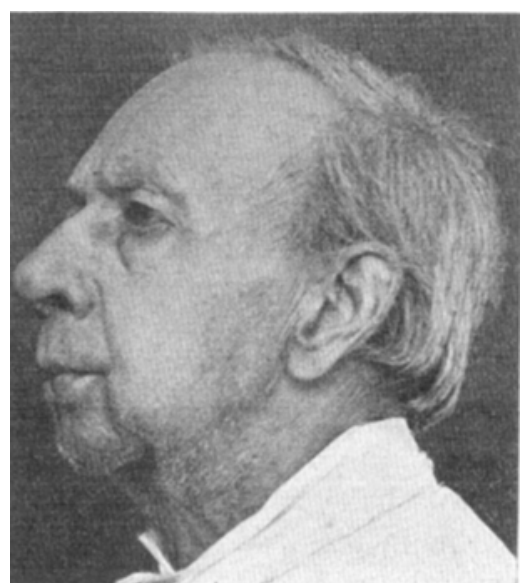

Fig. 1.

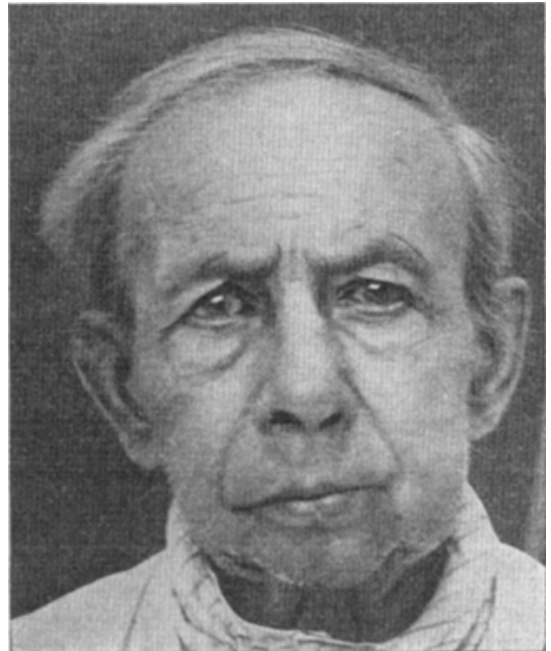

Fig. 2.

Care.-Mundboden a. Zunge,

Resektion des Mittelstucks des Coterkiefers.

Es ist klar, daß man, um den Kiefer richtig zn fixieren, einen Fremdkörper in den Mund einlegen muß und man wollte dies vermeiden, solange die Wunde noch von der Mundhöhle aus zugänglich und infizierbar war. Es wurde also die bereits eingetretene Verschiebung mühsam korrigiert, ehe der Apparat eingelegt wurde. Auch heute noch wird nach dieser Methode hier und da verfahren.

Aus später zu erörternden Gründen halten wir dieses Vorgehen heute höchstens noch für einen Notbehelf und wenden uns gleich den Methoden zu, welche direkt von der Operation ab den Kiefer in gesicherter Stellung erhalten wollen. Als hervorragendes Mittel müssen wir da die vom Zahnarzt Sauer in die Prothesenbehand- 
lung eingeführte "schiefe Ebene" bezeichnen. An dem erhaltenen Alveolarrande des Unterkiefers ist eine die Zähne umklammernde Prothese angebracht, an der Außenseite steigt neben den Zähnen eine schräg lateral aufwärts gerichtete Metallscheibe empor, die bei geschlossenem Mund bis zur Umschlagfalte der Oberlippe reicht. Bei Schluß und Öffnen des Mundes gleitet diese Platte neben den Oberkieferzähnen der gesunden Seite und macht ein Hinüberziehen des Unterkiefers nach der kranken unmöglich.

Diese Sauersche schiefe Ebene, welche bei bereits eingetretener Vernarbung die beste Hilfe ist, wird in der v. Bergmannschen Klinik, an deren Kranken sie Sauer zuerst anlegte, ausschließlich verwendet. Nach einem vor der Operation gefertigten Abdruck wird vom Zahnarzt der Alveolaraufsatz mit der Ebene gearbeitet, in Verbindung mit einem soliden Ansatzstück, welches in die Defektstelle reicht und so auch das Einsinken der Wange etwas verhïtet. Eine Dislokation des Kiefers ist mit diesem Apparat, soweit die schiefe Ebene ansreicht bei der Bewegung, ausgeschlossen.

In prinzipiell anderer Weise hat Claude Martin alle Folgen der Unterkieferexartikulation zn vermeiden gesucht, indem er sofort bei der Operation ein dem soeben entfernten Kieferteil nachgebildetes Ersatzstück in den Defekt einfügte, dessen Lage er dadurch sicherte, daß er es einerseits mit dem Kieferstumpf verband, andererseits direkt in die leere Gelenkpfanne stellte. Als Ersatzmittel nahm er Kautschuk, dasselbe war zum Reinigen mit einer Rinne versehen. Ein in dieser Weise ergänzter Kiefer muß, da das Ersatzstück zwischen der Gelenkpfanne und dem Kieferstumpf in Spannung erhalten wird, in korrekter Stellung bleiben und er muß bei richtiger Ansführung denjenigen Halt bieten, welcher zum festen Zubeißen notwendig ist. Dieses Verfahren, welches nur wenig Anhänger in Deutschland gefunden hat, ist seither einigemal modifiziert worden. Vor allem wurde an dem verwendeten Material Anstoß genommen, das durch Metall von Stoppany und später von Fritzsche ${ }^{1}$ ) ersetzt wurde. Auf des Letzteren in dieser Zeitschrift erschienene Arbeit verweise ich, eine gute Orientierung über die Prothesenfrage erlaubt ferner das Buch von Karl Witzel. ${ }^{2}$ ) Weitere, wie mir scheint zweck-

1) Fritzsche, Über Unterkieferprothesen und über einen neuen künstlichen Unterkiefer. Deutsche Zeitschr. f. Chir., Bd. 61, S. 560.

2) Karl Witzel, Chirurgie und Prothetik bei Kiefererkrankungen. Berlin 1905. Berlinische Verlagsanstalt. 
mäßige Verbesserungen hat dann der Greifswalder Zahnarzt Professor Schroeder ${ }^{1}$ ) ausgearbeitet. Nach des letzteren Angaben bin ich im wesentlichen vorgegangen, in Übereinstimmung mit Herrn Zahuarzt Roloff in Altona, dessen geschickter Arbeit wir den wesentlichen Teil des nun zu schildernden treftlichen Erfolges zuzuschreiben haben.

Unsere 40jähr. Patientin Miß Br. bemerkte im März 1906 an der Stelle, wo ihr vor 8 Jahren der linke untere Weisheitszahn gezogen wurde, eine kleine schmerzhafte Anschwellung. Im Juli 1906 suchte sie deshalb ihren Zahnarzt, Herrn Roloff, auf, derselbe machte eine kleine Incision und eröffnete eine kleine, im Fach des ehemaligen Weisheitszahnes gelegene Höhle. Am Tage darauf sah ich die Patientin; am Kiefer war von außen weder durch das Auge noch das Gefühl etwas Abnormes zu konstatieren. Der linke II. Molar im Unterkiefer war ein wenig lose, hinter ihm geriet eine' Sonde tief in den Unterkiefer hinein durch ein Loch, das von granulationsähnlichem Gewebe umgeben war. Der Zahn wurde extrahiert, aus dem Unterkiefer wurden mit scharfem Löffel geschwulstartige Massen herausgekratzt.

Die mikroskopische Untersuchung dieser Stücke zeigte ein zierliches Durcheinander von Hohlraumen und Schläuchen, die von zylindrischem Epithel umgeben waren, an dasselbe schlossen sich mehr kubische Zellen. Das Ganze bot das Bild der vom Schmelzorgan ausgehenden cystischen Epitheliome, die auch als Adamantinom bezeichnet werden.

Diese Geschwülste sind destruierend, von lokal malignem Charakter, und aus dem Studium der Literatur ergibt sich, daB sie auch rezirlivieren können. ${ }^{2}$ ) Ich beschloB daher, da die Zerstörung nach hinten bis in den Ramus ascendens mandibulae ging, die ralikale Operation unter Wegnahme des Kiefers bis ins Gelenk. Ich bemerke übrigens, dab, wie das Präparat später ergab, die teils solide, teils cystisch erweichte Geschwulst den Knochen medialwärts bis auf Pergamentdünne zerstört hatte, und daß am Übergang der Alveolarschleimhaut zum Tumor sich carcinomverdächtige Stellen mikroskopisch nachweisen lieBen.

Am 30. VII. 1906 machte ich einen Schnitt unterhalb des linken Unterkieferrandes, in der Nähe der Höhe des Zungenbeins entsprechend, unterband von hier aus die A. carotis ext., praparierte die linke Unterkieferhälfte bis in die Mundhöhle hinein frei, extrahierte den I. Molarzahn und durchsägte den Unterkiefer in der Höhe desselben, befreite ihn von seinen Muskeln und exartikulierte ihn. Ich habe dann - ein falsches Vorgehen, das ich später korrigierte - die Mundschleimhant wieder vernäht und dann das dem Schröderschen Hartgummimodell entnommene, von Herrn Roloff nach dem soeben entfernten Unterkieferstück abgepaBte Ersatzstück aufwärts in die Fossa glenoidalis gestellt und durch Vermittlung

1) Schroeder, Beitrag zur Unterkieferresektionsprothese. Odontolog. Blätter X, Heft 3.

2) Hardouin, Bull. et mémoir. de la société de Paris, 1904. Ref. Hildebrands Jahresbericht f. Chir. für 1904. 
des später zu beschreibenden Metallstreifens an den Unterkieferrest angenäht. Die äuBere Wunde wurde z. T. tamponiert, z. T. genäht.

Der Verlauf war ungestört, Patientin konnte den Mund öffnen und schließen. Am 3. VIII. 1906 nahm ich in Narkose die Prothese wieder heraus, trennte die vernähte Schleimhaut, kürzte das mediale Unterkieferstück noch um etwas und nähte eine neue entsprechende Hartgumminachbildung an den Kiefer an, das andere Ende wieder ins Gelenk stellend. Diesmal wurde, soweit es ging, die Schleimhaut unter, also nach auBen, von der Prothese vernäht. Wieder Tamponade eines Teiles der Wunde. (Ich wiederhole, daB dieser Akt nur durch das nicht ganz richtige Vorgehen am 30. VII. nötig wurde.)

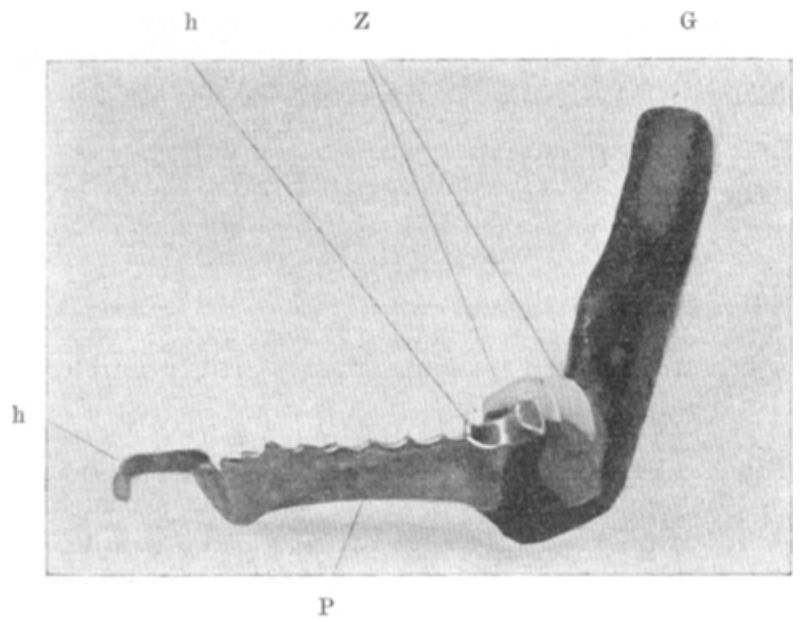

Fig. 3.

Definitive Prothese, in der Mitte an der Platte die Haken zur Fixation an den Zähnen, dann zwei künstliche Zảhne, oben der ins Gelenk reichende Fortsatz.

$G=$ Gelenkteil. $\quad \quad \quad P=$ sublinguale Platte. $\quad \mathrm{Z}=$ künstliche Zähne. $\mathbf{h}=$ Haken zum Festhalten an den gesunden Zähnen.

Temperatur einmal $38,2^{0}$, sonst höchstens $37,8^{\circ}$. Täglich mehrmals wird die Prothese mit Borlösung und Wasserstoffsuperoxyd ausgespült, noch häufiger die Mundhöhle. Tampon täglich gewechselt. Zuerst floB ein Teil der Spülflüssigkeit zum Munde heraus, dies hörte etwa vom 10. VIII. an auf; es erschienen gute Granulationen. Die Stelle vor dem Ohr sowie am Stumpf zeigte leichte Schwellung und Druckempfindlichkeit.

17. IX. wird in Narkose durch Entfernung der Drahtnaht die Prothese herausgeholt und an ihrer Stelle eine definitive eingesetzt, deren oberer Teil ins Gelenk reicht, der untere trägt eine sublinguale Platte mit je einem proximalen und distalen Haken zum Halt an den Zähnen (Fig. 3).

Diese Prothese wird von da ab täglich herausgenommen, gereinigt und wieder eingesetzt. Patientin wird am 21. IX. entlassen in die Behandlung des Zahuarztes. 
Anfang November ${ }^{1}$ ) war der Befund folgender: ÄuBere und innere Wunde sind ohne Fistel verheilt, die Narbe nur bei Emporheben des Gesichts sichtbar. Keine seitliche Verschiebung des Kiefers, kein Einsinken der Wange. Mund kann auf mehrere Zentimeter geöffnet und ganz fest geschlossen werden. Patientin iBt alle Speisen wie früher (z. B. Brot nebst Rinde mit Schinken), kann fest kauen. Es bestehen keine Schmerzen, die früher bestandene Schwellung ist verschwunden. Sie setzt sich selbst die Prothese mit den künstlichen Zähnen aus und ein, den Kolben ins Gelenk stellend, dann die Platte niederdrückend und die Haken der Platte an den Zăhnen fixierend.

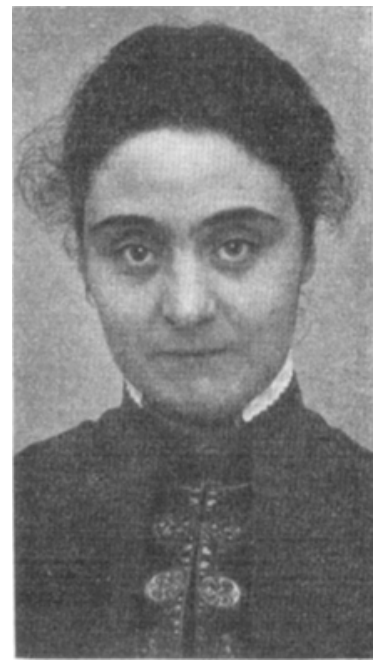

Fig. 4.

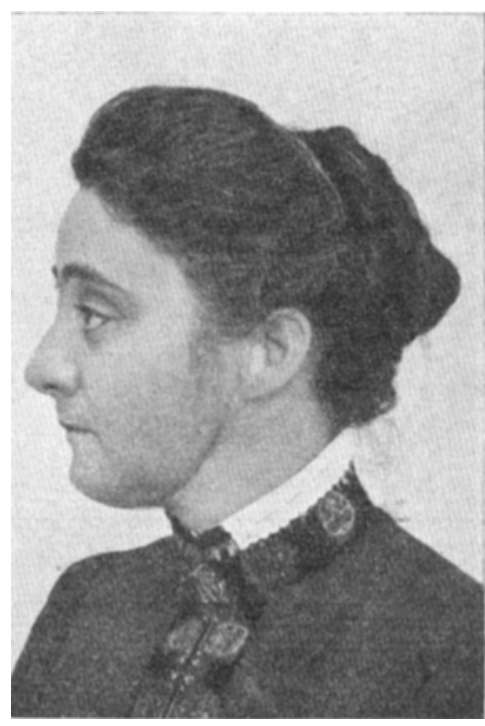

Fig. 5 .

Exartikulation des linken Lnterkiefers.

29. XII. Zustand unverändert. Morgens soll neben der Prothese noch angesammeltes Sekret kommen. Innere Wunde, so weit sichtbar, epithelisiert; der ins Gelenk gehende Schleimhauttrichter läßt eine Sonde noch auf etwa $5 \mathrm{~cm}$ eindringen.

Die Vorteile der hier angewandten Prothesenbehandlung nach den auf Claude Martins Prinzipien basierenden Angaben Schroeders sind für jeden, der ein solches Resultat gesehen hat, außer allem Zweifel. Kosmetisch ist der Erfolg, da das Oval des

1) Vorgestellt im ärztl. Verein zu Hamburg am 13. XI. 1906. 
Gesichts so ganz erhalten bleibt, ein vollkommener; Unbefangene kommen gar nicht auf den Gedanken, daß hier im Mund etwas nicht in Ordnung ist. Der Erfolg ist auch vollständiger wie mit der Sauerschen Prothese, die Formen des Gesichts werden absolnt er-

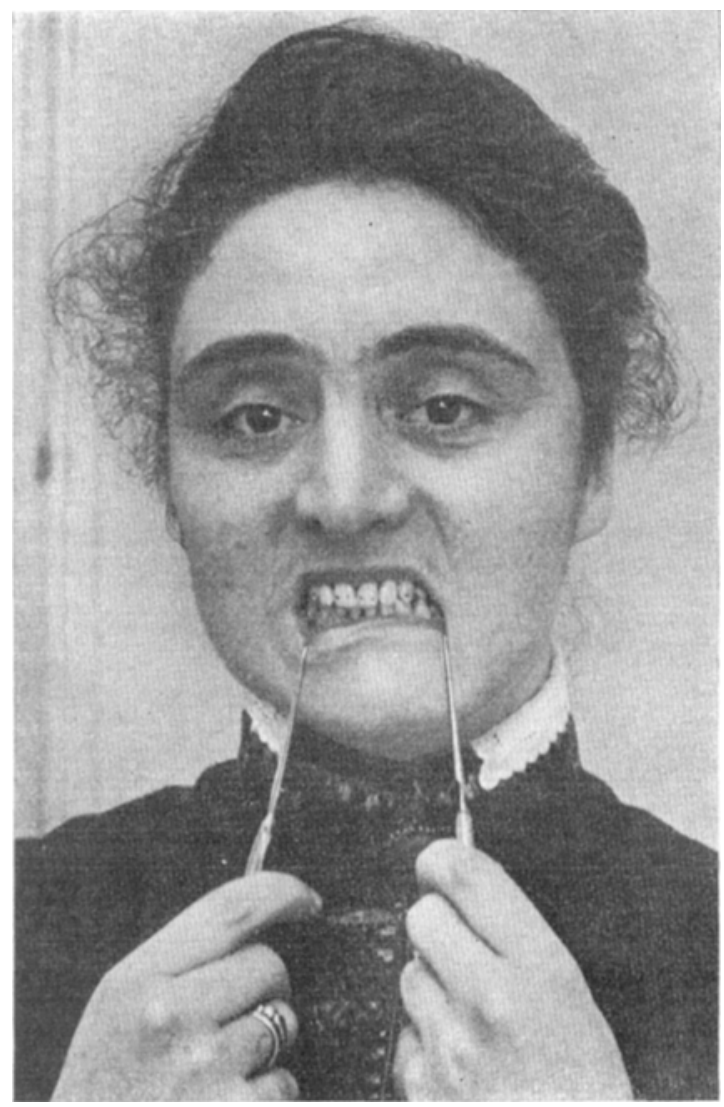

Fig. 6.

Linksseitige Unterkieferexartikulation, Zahnschluß mit Prothese nach Schroeder.

gänzt und wir haben beim Öffnen des Mundes nicht den merkwürdigen Anblick der Metallplatte im Munde. Die Kaufähigkeit ist außerordentlich gut. Allerdings muß dazu der Vorteil der Martinschen Methode ganz ausgenutzt werden, insofern das obere Ende des Ersatzes direkt in die Fossa glenoidalis eingestellt wird. Das scheint mir nicht immer durchgeführt zu sein; Til- 
$\operatorname{mann}{ }^{1}$, welcher mit Schroeder gearbeitet hat, sagt, daß das obere Ende zwischen Pterygoidei und Masseter eingesetzt werde, und „durch den schweren Metalleinguß werde erreicht, daß die Hülse fester und sicherer gelagert sei." (also doch nicht durch die beiden festen Punkte: Gelenk einerseits, Kiefernaht andererseits), und „biete sie gleichzeitig den sich bildenden Granulationen und den umliegenden Weichteilen ein dem entfernten Knochenstück ganz und gar entsprechendes massives Modell, um das sie sich herumlagern können." ?) Auch in dem ersten Fall (3) von Fritzsche kann die Prothese ihrer Form nach nicht zum Einstemmen ins Gelenk benutzt sein, während Fr. im Fall 4 dies ausdrücklich betont. Dies hervorzuheben halten wir für um so wichtiger, als die Neigung des Kiefers, nach der operierten Seite hinüberzudrängen, eine so große ist.

Schon wenn die Prothese anf kurze Zeit ans dem Munde entfernt war, macht sie sich bemerkbar, und trat auch in unserem Fall in der ersten Zeit gelegentlich hervor, wo offenbar die Fixation am Resektionsende durch die Drahtnaht noch wackliger war wie später an der Gebißplatte. Damit wird wohl auch folgendes zusammenhängen. Patientin hatte bei längerem Sprechen in den ersten Wochen öfter einen plötzlichen Schmerz und der Kiefer stand für einen Augenblick still; sie konnte das aber durch eine jähe Bewegung mit dem Kiefer wieder beseitigen. Ich nehme an, daß hier der Zapfen subluxierte und sie die Prothese durch die Bewegung wieder einrenkte. Der Halt zwisehen den beiden festen Punkten ist also für ein erfolgreiches Arbeiten der Einlage notwendig, und wenn Tilmanns Patientin nicht kauen konnte, so muß man annehmen, daß dieser Halt fehlte. ${ }^{3}$ ) - Der letzte Vorzug ist der, daß durch diese Anbringung dem Kiefer, der sich also auch auf der operierten Seite anfs Gelenk lehnt, mechanisch ein Teil seiner Arbeit abgenommen wird, während diese bei Sauers Prothese die an der defekten Hälfte durch nichts geschützte gesunde Seite allein

1) Tilmann, Über Unterkieferresektionen. Deutsche med. Wochenschr. 1903, Nr. 23.

2) Schroeder legt auf die Artikulation gegen den Oberkiefer das Hauptgewicht, hält aber dazu das Gelenk direkt für unnötig.

3) Schroeder bildet seinen Ersatz aus Hartgummi in der Tat ohne Proc. condyloideus: die Höhle soll durch Granulationen ausgefüllt werden (siehe Schroeder). Die Anwendungsweise zahnärztlicher Prothetik im Bereiche des Gesichts mit besonderer Berücksichtigung des sofortigen Kieferersatzes nach Resektion. Greifswald. S. 59 u. 61. 
besorgt. Die dadurch entstehende Ermüdung ist begreiflich. Anch bei unserem Vorgehen tritt sie ein, und man muß durch methodische Übungen dem Kiefer allmählich größere Arbeit zumuten. Auf diese Weise haben sich in unserem Fall die Ermüdungsgefühle sukzessive veringert.

Von den Bedenken, welche diesen Apparaten entgegenstehen, will ich das letzte vorwegnehmen. Wird die Prothese dauernd ihre Brauchbarkeit behalten? Es ist den Martinschen Einlagen schon wiederholt vorgeworfen worden, daß sie die Zähne lockern, an welchen sie sich festhalten. Dazu ist also ein regelmäßiges Kontrollieren durch den Zahnarzt erforderlich, und, wenn das Ereignis eintritt, eine Änderung der Fixation, welche entweder an anderen Zähnen stattfinden oder in Kappenform den Kiefer umgreifen muß. Das sind individuelle Verhältnisse, denen der geschickte Zahntechniker sich anpaßt. Die andere Frage ist die, ob das Gelenk es auf die Dauer ertragen wird, daß der Fremdkörper in offener Mundhöhle in ihm artikuliert. Nun, eine Analogie dafür gibt es allerdings meines Wissens nicht, und da ich die offenbar allerdings nur spärlichen Fälle nicht auf ihr Dauerresultat untersuchen kann, bei denen gleichartig vorgegangen wurde, so kann man nur Vermutungen hegen. Im günstigsten Falle wird ja der Knorpel atrophieren und eine harte Narbe den Kolben tragen. Sollte es aber zu einer Störung kommen, so wird man bei dem erreichten guten Resultat sehr gut den Ramus ascendens der Prothese um etwa $1 \mathrm{~cm}$ verkürzen und für die kurze Zeit die Verschiebung des Kiefers etwa noch im Notfall durch Hinzufügen einer schiefen Ebene hintanhalten, bis das obere Ende des Trichters geschrumpft ist und die Gelenkpfanne zndeckt. Sobald dies erreicht ist, wird dann der Zapfen wieder festen Widerhalt finden, ohne das Gelenk zu treffen. ${ }^{1)}$ Übrigens möchte ich mich bedingt der Ansicht von Herrn Roloff anschließen, daß nämlich die Prothese auch durch die sämtlichen außerordentlich straff sich anlegenden Weichteile mit in Spannung erhalten wird, so daß dadurch ein Teil des Druckes vom Gelenk genommen wird. Die Spannung ist ja so stark, daß man nicht lange die Prothese entfernen darf; bei längerem Herauslassen würden die Weichteile sich so eng zusammenschließen, daß man den Bolzen kaum wieder hineinbrächte. Es ist denkbar, daß

1) Der Mechanismus würde dann der gleiche sein, den Schroeder jetzt mit seinen ausgehöhlten Hartgummischalen ohne Proc. condyloideus erreichen will. (Inzwischen mit vollem Erfolg ausgeführt. Nachtrag bei der Korr.) 
durch diesen Mechanismus die Gelenkfläche in einer Weise entlastet wird, welche ihr Erhaltenbleiben ermöglicht.

Ehe ich auf die übrigen Bedenken eingehe, muß ich hervorheben, daß die Behandlung mit diesen Apparaten eine gewisse Willensstärke und Intelligenz bei dem Kranken voraussetzt. Bei hysterischen, stark neurasthenischen Menschen wird es großer Geduld bedürfen, wenn die definitive Prothese täglich zum Reinigen aus- und eingesetzt wird, und ich kann mir wohl denken, daß daran die Sache scheitern kann. Zudem muß der Kranke doch selbst lernen, die Prothese herauszunehmen und einzulegen. Es war eine Freude, zu sehen, mit welchem Geschick unsere Kranke dies vor dem Spiegel, sogar unter den Augen einer großen Ärztekorona vollführte.

Das größte Bedenken, welches von jeher gegen die Immediatprothese vorgebracht wurde, welches wie ich aus einer Reihe von Antwortschreiben sehe, noch jetzt seine Kraft hat, und auch mich vor dieser Beobachtung beseelte, ist das: ob die Einführung eines so großen Fremdkörpers in eine so bedeutende Wunde nicht eine unverantwortliche Gefahr birgt. Wir können ja die Wundhöhle gegen den Apparat und gegen die Mundhöhle nicht völlig abschließen. Bei der Kontinuitätsresektion wird dies ja vielfach durch das Vernähen der Schleimhaut über der Ersatzschiene, welche einheilen soll, angestrebt und in diesem Sinne ist auch der Berndtsche Zelluloidring bei mehreren, leider nicht veröffentlichten Fällen der Helferich schen Klinik in Greifswald (mündliche Mitteilung) und von Dr. Berndt in seiner eigenen Tätigkeit verwendet worden. ${ }^{1)}$

B. beschreibt, wie ein entsprechend zurechtgebogener Zelluloidring für das verlorengegangene Kieferstück in die Wunde eingelegt, die Mundhöhle durch Naht sorgfältig abgeschlossen wurde. Die Zellnloidprothese soll in der Wunde ohne Fistel einheilen, den Kiefer gerade halten, und Öffnen und Schließen des Mundes gestatten.

Indem ich den Wunsch hatte, zunächst wenigstens ähnlich sicher zu gehen, vernähte ich sofort nach Einnähen der Prothese die Schleimhaut nach einwärts davon, was selbstverständlich in weiterem Umfang gelang. als wenn ich sie sofort unter, nach außen davon vernäht hätte, wobei die Spannung größer ist und aufwärts eine Lücke bleiben muß. In der zweiten Sitzung habe ich das dann korrigiert, denn nur bei letzterem Vorgehen ist das MartinSchroedersche Verfahren durchführbar. Nun, wir wissen ja, daß

1) Siehe weiter unten noch die Mitteilung von Garré. 
im ganzen eine Infektion von noch so großen Operationen im Mund selten ist; ich erinnere nur an die ausgedehnten Plastiken nach Dieffenbach, bei welchen doch in der Regel nur unbedeutende Nahteiterungen vorkommen. Daß die Sache durch den „Fremdkörper" so sehr viel anders werde, kann ich nicht glauben; denn schließlich ist eine ausgehöhlte Hartgummiprothese kaum anders wie ein Drainrohr aufzufassen. Die Einrichtung dieser Prothese erlaubt wirklich auch eine ausgezeichnete Drainage durch häufige Spülung, auf deren Bedeutung schon hingewiesen wurde.

Aber eins muß doch verlangt werden, daß nämlich die äußere Wunde wenigstens zum Teil offen gelassenwird. Imobersten Teil, da wo die Prothese zwischen den Muskeln ins Gelenk geht, kann man die Schleimhaut nicht vernähen, immer bleibt hier eine offene Verbindung zwischen Wunde und Mundhöhle. Eine Infektion, die sich hier festsetzt - ihr Ausbleiben können wir nicht garantieren - muß sich, den Muskeln folgend, in die Wundhöhle senken, ja schon die Spülflüssigkeit kann hier eindringen; und wenn dann keine Phlegmone entsteht bei völligem Wundverschluß, dann ist das nicht unser Verdienst. Auch am Kieferende kann die Schleimhaut nicht sicher abgeschlossen werden, und hier ist ein Ventil um so notwendiger, als der dem Knochen anliegende Apparat mitsamt der Drahtnaht Entzündung machen kann. Wenn die Wunde offen ist, wir den Tampon wechseln können, dann können wir auch den Sekreten Abfluß sichern, ohne das geben wir uns auf gefahrvollen Boden.

Bis zu einem gewissen Grade gilt das auch für die Kontinuitätsresektionen, wenn wir bei ihnen die Schiene unter die darüber vernähte Schleimhaut lagern. Auch hier bleibt immer am Resektionsende eine Stelle, wo die Naht nicht ganz dicht sein kann. Von dieser Stelle kann stets eine Infektion der den Fremdkörper beherbergenden Wunde ausgehen und sie wird auch der Grund sein, weshalb die volle Einheilung so oft nicht gelingt.

Über die Nachbehandlung der Unterkieferoperationen an 30 Anstalten habe ich mich zu informieren gesucht, nämlich an sämtlichen deutschen und beiden Wiener Universitätskliniken, endlich über einige große Krankenhausabteilungen. Eine Anzahl von ihnen konnte über keine Erfahrungen berichten, weil keine einschlägigen Fälle vorgekommen waren. Dagegen läßt sich ein Urteil über das Vorgehen an einigen zwanzig Instituten aus den Antworten gewinnen. 
Bei den Kontinuitätsresektionen sind von der überwiegenden Mehrzahl der Chirurgen künstliche Mittel verwendet worden, nur an einer Universitätsklinik scheint man sich auch hier anf die Wundbehandlung beschränkt zu haben. Sehr pessimistisch lauten drei Antworten, welche besagen, daß Versuche mit sofortigem Einlegen von Schienen oder Draht gemacht wurden, von denen die Betreffenden nicht befriedigt waren. Gerade bei gutartigen Tumoren ist es sehr bedauerlich, wenn die Nachbehandlung versagt nnd Poppert (Gießen), welcher zwei hierher gehörige Operationen ausführte, beklagt es sehr, daß ihm eine geeignete zahnärztliche Hilfe nicht zur Verfügung stand.

In 19 weiteren Anstalten wurden sofort nach der Operation die Resektionsenden durch Schienen miteinander verbunden, welche im ganzen nach flem Hansmann-Partschschen Prinzip gearbeitet waren. Nur in Würzburg wurde dies wegen Wundstörungen zugunsten sekundärer Prothesen wieder aufgegeben. In den übrigeu Instituten war das weitere Vorgehen insofern ein verschiedenes, als 7 versuchten, die Schienen zum Einheilen zu bringen, während 11 die Schiene von vornherein nur als provisorisch ansahen, um sie später durch eine zahnärztliche Prothese ersetzen zu lassen.

Betrachten wir zunächst, was bei den Versuchen, die Schiene einheilen zu lassen, heransgekommen ist. Ohne Zweifel wäre es das Beste, wenn man das verloren gegangene Kieferstück durch einheilende Körper dauernd ersetzen könnte, vor allem wären wir unabhängig vom Lauf der Dinge, welcher durch Ausfallen der Zähne auch der schönsten Prothese schließlich ihre Festigkeit nimmt, und von dem Umstand, daß mancher Kiefer schon vor der Operation zalınlos ist. Zwei Institute (Heidelberger und Rostocker Klinik), geben nun schlechtweg an, daß die Einheilung der Schienen auf die Dauer mißlang, so daß sie, auch wenn der Verlanf zunächst glatt war, später entfernt werden mußten. Wie Herr Dr, Völcker (Heidelberg) schreibt, wurden bei Czerny Versuche mit der Einheilung von Hartgummi- oder Kantschukprothesen, von Silberdraht, auch von frischem sterilisiertem Tierknochen gemacht: sie mußten stets nach kürzerer oder längerer Zeit entfernt werden. Gleiche Erfahrung scheint nach der Mitteilung von Herrn Dr. Ranzi in früheren Versuchen von Eiselsberg gemacht zu haben. Wechselnd war der Erfolg in Breslau (bezw. wohl Königsberg), Halle, München. Garré hat sich, wie er mir freundlichst mitteilt, der Partschschen Schiene bedient und ist mit den Erfolgen sehr zufrieden; eine Fistel bleibe wohl bei Resektion von viel Weichteilen 
zurück. Der in Halle bei v. Bramann verwendete Metallbügel wird, nach der Mitteilung von Haasler, an die Kieferenden angenäht und heilt in der Regel glatt ein, während dies nach Gebeles Auskunft in der v. Angererschen Klinik in München in mehreren Fällen nicht eintrat. An der v. Brunsschen Tübinger Klinik (von Brunn) und bei Rehn in Frankfurt (Nötzel) heilte die Partschsche Schiene glatt ein, doch hat es sich beide Male nur um 1 Fall gehandelt.

Nach dieser immerhin ausgedehnteren Übersicht können wir nie darauf zählen, daß der Organismus, auch wenn wir die Schleimhaut weit genug über der Schiene vernähen können, sich den Fremdkörper einverleibt. Bei Hartgummiprothesen am wenigsten, wie aus mehreren Mitteilungen hervorgeht, am ehesten offenbar bei Metallschienen. Man ist wohl berechtigt, ihre Anwendung vorzuziehen, wenn der Mund keine Zähne hat, oder an das Anlegen einer Prothese durch den Zahnarzt nicht zu denken ist, weil es sich um arme, oder wenig einsichtige Individuen handelt. Man muß dann aber mit der Möglichkeit einer Fistel rechnen, welche weder schön noch ganz gefahrlos ist. Immerhin, genützt hat man dem Kranken auch dann, wenn man die Prothese später entfernen muß: denn sie hat bis dahin die Dislokation der Kieferhälften verhütet und eine Prothese ist auch jetzt noch anzubringen.

Dagegen wird Eiterung und Fistelbildung auszuschließen sein, wenn der einzulegende Ersatzteil nicht in dauernder Berührung mit der Wunde bleibt. Dies geschieht, indem zwar zunächst auch die Schiene, welche die Kieferteile aneinander hält, an letztere angenäht wird, jedoch mit der Absicht, später entfernt zu werden, nachdem die unterhalb der Schiene zusammengenähte Schleimhaut vereinigt ist. Dann wird ein vom Zahnarzt gearbeiteter Ersatz in den Defekt gefügt und mit geeigneten Vorrichtungen an den Zähnen des Alveolarrandes befestigt. Derselbe sitzt im Munde herausnehmbar, wie eine Gebißplatte.

In dieser Art wird an den Universitätskliniken von Berlin, Bonn, Göttingen, Greifswald, Kiel, Leipzig, Nünchen, Wien I, sowie an den Krankenhausabteilungen von Hannover, Köln und Altona verfahren. Läßt sich die Prothese gut befestigen, so ist das die beste Methode, mit dem Apparat können sofort die verloren gegangenen Zähne durch künstliche ersetzt werden.

Wir müssen hier noch erwähnen, daß die zahnärztliche Hilfe auch dann noch Erfolge erzielt, wenn der Patient nach der Resektion jahrelang ohne Apparat gegangen ist. Die spitzwinklig nach 
innen zusammengezogenen Kieferstücke auseinander zu bringen, gibt es schon eine Reihe von Verfahren. ${ }^{1}$ ) Herr Privatdozent Dr. Hentze-Kiel hatte, um einen praktischen Beweis anzuführen, wie er mir schreibt, einen Knaben zu behandeln, bei dem vor Jahren das Mittelstück des Unterkiefers wegen Sarkom in breiter Ausdehnung reseziert war: zwei angefertigte Ersatzstücke waren nie getragen worden. Es gelang Hentze, die völlig nach innen stehenden narbig fixierten Unterkieferstümpfe in sinnreicher Weise durch die Kraft stetig stärker gewählter Stahlfedern innerhalb von acht Wochen auseinander zu sprengen und dann durch eine sorgfältig gearbeitete Prothese in guter Stellung zu erhalten. (Abbildungen in der Mitteilung von Kleinm an n, Korr.-Blatt für Zahnärzte. Bd. 34, Heft 4, S. 327 ff. Okt. 1905.)

Zur Entscheidung der Frage für die Nachbehandlung der Exartikulation schrumpft die Zahl der verwertbaren Antworten zusammen. Acht bedeutende Kliniken und zwei große Krankenhausabteilungen haben keine Erfahrungen sammeln können. In vier Instituten hat der Mangel an hinreichend durchgebildeten Zahnärzten die Möglichkeit der Prothesenbehandlung nicht zugelassen. Drei Kliniken und eine chirurgische Abteilung sehen von jeder Prothese $a b$; von zwei Seiten wird angegeben, daß man trotzdem mit dem Erfolg ziemlich zufrieden gewesen sei. Das ist natürlich nur bei niedrigen Ansprüchen möglich.

An im ganzen 11 Anstalten wurden Apparate verwandt. Es ist bezeichnend, daß hierron nur zwei diese sekundär, nach erfolgter Heilung eingelegt haben, beide nachdem sie mit Immediatprothesen keinen rechten Erfolg gehabt hatten. Dieses unbefriedigende Resultate bezog sich in Heidelberg darauf, daß die Versuche, Ersatzstücke einheilen zu lassen, gescheitert waren. Ob das an der zweiten, der Würzburger Klinik, beabsichtigt war, vermag ich nicht sicher zu entscheiden, es scheint sich vielmehr um provisorische Immediatprothesen gehandelt zu haben. Herr Dr Burckhardt schreibt mir. daß bei sofortigem Einlegen mehrfach „die Wundheilung gestört war, wohl infolge des mechanischen Reizes, und die Prothesen mußten häufig geändert werden". In Würzburg werden dann die aus vulkanisiertem Kautschnk vom zalnärztlichen Institut gefertigten Prothesen nach einigen Monaten eingesetzt und auf diese Weise befriedigende kosmetische und funktionelle Resultate erzielt. Bei den Heidelbergen Fällen wurde mit den se-

1) Siehe Karl Witzel, 1. c., S. 205. 
kundären Prothesen ein eigentlich befriedigender Erfolg nicht erreicht.

Wenn Patienten mit schon verheilter Exartikulation zu uns kommen, so müssen wir die Verstellung des Kiefers korrigieren und durch eine mit "schiefer Ebene" verbundene Prothese die Zahnreihen reponiert erhalten. Stehen wir aber noch vor der Operation, so versuchen wir stets durch eine der gleich zu besprechenden Immediatprothesen die Verschiebung des Kiefers ein für allemal zu verhüten. Von vornherein auf eine sekundäre Prothese hinausgehen, heißt resignieren.

Die an den befragten Instituten gebranchten, sofort eingesetzten Apparate kann man mehrfach gruppieren. Der einfachste äußerst sinnreiche ist die schiefe Ebene Sauers. Daß man mit ihr jede Verschiebung vermeiden kann, habe ich neuerdings wieder an einer Patientin der v. Bergmannschen Klinik am 17. XII. 1906 gesehen. Das einige Monate vorher von Exz. v. Bergmann halbseitig exartikulierte Mädchen zeigte keine Dislokation der Knochen, konnte den Kiefer weit öffnen, ohne daß Verschiebung eintrat und fest in gerader Richtung beißen.

Es ist leicht, an diese schiefe Fbene einen Fortsatz anzubringen, welcher in ein größeres Ersatzstück ausläuft, das die entstandene Defekthöhle etwas füllt und die neuen Zähne trägt. Bei diesem von $\mathrm{Hahl}$ stets ausgeübten und auch von andern empfohlenen Verfahren wird auch dem Einsinken der Wange etwas entgegengewirkt; es war das typische Verfahren an der Klinik meines Lehrers v. Bergmann ${ }^{1}$, die Schleimhaut wird dabei nach sofortigem Einlegen der vorher angefertigten Prothese außerhalb der letzteren möglichst vollständig vereinigt. Nach dieser Weise arbeiten, wie ich den Angaben entnehme, anscheinend noch zwei andere Kliniken; hier verhindert also immer die Sauersche schiefe Ebene die Dislokation des Kieferrestes.

Im Gegensatz dazu stehende Immediatprothesen, welche einen möglichst vollständigen Ersatz des entfernten Knochens erstreben, und die Stellung sichern durch die Fixation am Kieferstumpf einerseits, durch das Aufstemmen aufwärts in der Richtung der Gelenkpfanne andererseits, sind in acht Anstalten verwendet worden.

Wir müssen zunächst gesondert die Methode besprechen, welrhe

1) Gösta Hahl, Die Prothesen nach Kieferresektionen. Deutsches Arch. f. klin. Chir., Bd. 54, H. 4. 
nach seiner eigenen Mitteilung Garré seit Jahren anwendet. Er benutzt „als Prothese einen einfachen umgebogenen dicken Draht (Klavierdraht) - der mit dem bleibenden Unterkieferknochen verankert wird. Die abgebogene Stelle artikuliert schmerzlos im Gelenk". Nach der Beschreibung muß ich annehmen, daß dieser Draht, in die Wunde gelegt, überall intrabuccal verläuft. Herr Geh.-Rat Garré schreibt weiter, daß er mit den Erfolgen sehr zufrieden sei - selbst wenn eine Fistel zurückbleibt, sei das Resultat ein recht gutes.

Der Rat, Silberdrat als einzuheilenden Ersatz am Unterkiefer zu verwenden, wurde anf Grund einer Operation bei osteomyelitischer Nekrose von C. Hofmann gegeben. (Centralbl. f. Chir. 1900, S. 1145.) Nach unseren Antworten ist das Verfahren bei Kontinuitätsresektionen mehrfach ohne befriedigendes Resultat verwendet worden. Um so erfrenlicher ist die Erfahrung Garrés, denn sie zeigt, daß man auf diese einfache Manier durch eine Danerprothese ohne Zahnarzt der Dislokation entgegenwirken kann, wenn auch wohl das Einsinken der Wange auf die Dauer nicht ganz ausbleiben wird. Die Möglichkeit aber ist von großer praktischer Bedeutung.

Es bleiben nun noch fünf Anstalten, an welchen bei Exartikulation des Unterkiefers nach den Claude Martinschen Principien vorgegangen worden ist. In Köln hat Bardenheuer die von ihm bei Kontinuitätsresektion gebrauchte Zelluloidspange in einem Fall von ausgedehnter Tuberkulose so verwandt, daß er den oberen, dem anfsteigenden Ast entsprechenden Teil aufwärts in die Wundhöhle stellte, die unteren 2\%: durch Daruntervernähen der Schleimhant extrabuccal lagerte und mit dem Kiefer vernähte. Wie ich seiner freundlichen Antwort entnehme, soll später eine Prothese eingelegt werden, welche am Unterkieferresektionsrand sich hält, und hinten mittels Feder eine Stütze am Oberkiefer gewinnt.

An der Bonner Klinik hat Schmieden eine Operation erlebt, bei welcher „eine interimistische Prothese bei einseitiger Exartikulation in der Weise angefertigt war, daß ein knopfartiger, dem Proc. articularis entsprechender Fortsatz in die Gelenkhöhle hineingestellt wurde. Vorn wurde diese Prothese mit Draht angenäht".

Den schon vor fünf Jahren vom Zahnarzt Fritzsche mit seiner Prothese versorgten Patienten Friedrichs in Leipzig, ferner (ler von Tilmann (Greifswald) operierten Patientin, bei welcher Schroeder seine Prothese verwandte, reiht sich unsere Mitteilung an. In analoger Weise, wurden an den von uns befragten Anstalten nur noch zwei Exartikulationen, und zwar nach Fritzsches 
Anweisungen nachbehandelt. Sie betreffen Sarkome des Unterkiefers aus der I. Wiener Klinik von Eiselsbergs, deren Prothesen von Dr. Pichler angefertigt wurden. (Wien. klin. Wochenschr. 1906, S. 1906). Wie Herr Dr. Ranzi schreibt, war "das kosmetische Resultat ein ideales, die Zahnreihen paßten vollkommen aufeinander und die Deviation konnte vollständig vermieden werden. Auch die funktionellen Resultate waren sehr befriedigend".

Auf die außerdeutschen Länder, auch auf die Schweiz, wo die Martinschen Prinzipien z. T. dank der tatkräftigen Förderung durch Stoppany, schon mehr Anerkennung gefunden haben, bin ich nicht näher eingegangen.

Aus unserer Übersicht geht hervor, daß sich die idealsten Resultate, mit Vermeiden jeder Deviation sowie jedes Einsinkens der Wange mittels der Verfahren nach Martin (Stoppany, Fritzsche, Schroeder) erzielen lassen. Diese Behandlung setzt aber einen etwas willensstarken und intelligenten Patienten vorans. Wenn Geh.-Rat Madelung schreibt, daß die Kranken diese Immediatprothesen nicht vertragen, so kann ich das wohl für manche verstehen. Und wenn er hinzufügt, daß sie sich nicht für "Unfallpatienten“ eignen und nur für einigermaßen Gebildete, so ist darin eine Einschränkung ausgesprochen, welche uns unter Umständen nötigen wird, auf die idealsten Erfolge zu verzichten.

Ich glaube, daß man bei Gebildeten, welche die etwas angreifenden Manipulationen bei dem täglichen Ein- und Auswechseln der Prothese nicht vertragen, zu der Methode der von Bergmannschen Klinik, der Sauerschen schiefen Ebenemit teilweisem soliden Ersatz des Kiefers, auf die man ja noch in jedem Stadium der Behandlung rekurrieren kann, seine Zuflucht nehmen soll. Bei solchen Kranken, bei denen wir voraussehen, daß sie für eine Prothese nicht dauernd geeignet sind ( - manche Menschen werfen ja nach der Entlassung überhaupt jede Bandage, Apparat oder Prothese in die Ecke -), können wir mit Garré die umgebogene Drahtschlinge zur dauernden Einheilung ins Gelenk stellen und mit dem Resektionstumpf verankern.

Dasselbe muß der tun, dem gar keine zahntechnische Hilfe, auch nicht durch nachträgliche auswärtige Konsultation, zur Verfügung steht. Unsere Erhebungen zeigen leider, daß sowohl in einigen Universitäten wie großen Städten die Zahnärzte sich nicht auf der Höhe befinden, um den hier in Frage kommenden, immerhin bedeutenden Anforderungen an Wissenschaft und Technik zu 
entsprechen. Hoffentlich werden wenigstens die UniversitätsKliniken weiterhin durch Anstellung völlig durchgebildeter Zahnärzte als Leiter der betreffenden Institute davor bewahrt, aus Mangel an technischer Hilfe von Prothesen Abstand nehmen zu müssen. Denn mag man auch noch so sehr wünschen, daß der Chirurg auch ohne Zahnarzt fertig wird: etwas nach jeder Richtung Vollkommenes wird er ohne ihn nicht erreichen.

Um sofort die richtige Schiene einsetzen zu können, muß man solche vorrätig haben. Sowohl zum Ersatz bei der Kontinitätsresektion wie bei der Exartikulation sind vorzüglich geeignet die Hartgummimodelle von Schroeder. ${ }^{1}$ ) Sie sind Nachbildungen des vollkommenen Unterkiefers, nur der Kronenfortsatz fehlt, der Gelenkfortsatz und Ramus ascendens ist zur Drainage ausgehöhlt. Sie werden in 3 verschiedenen Größen von der Firma Meyerholz in Hannover geliefert. An der Vorderfläche der Rami horizontales ist in eine Hohlkehle eine breite Metallschiene einzuschieben. Nach der Resektion wird ein entsprechendes Stück herausgesägt, und mittels der Metallschiene, welche über die stehengebliebenen Kieferenden hinüberreicht, mit Draht an dieser fixiert. Bei der Exartikulation kommt der obere Fortsatz direkt ins Gelenk. Um das Modellstück passend zurecht zu sägen und zu feilen, ist es erwünscht, außer Laubsäge und Feile einen Schraubstock zu haben. Die Desinfektion des Modells geschieht in Sublimatlösung.

Nach Erledigung der Prothesenfrage möchte ich noch ein paar Bemerkungen zur Ausführung der Exartikulation machen. Der Vorakt, welcher sie zu einer so sauberen Operation macht, ist die Unterbindung der A. carotis externa. Schon vor Jahren habe ich die Leistungsfähigkeit dieses längst von Kocher empfohlenen Eingriffes für die Oberkieferresektion hervorgehoben ${ }^{2}$ ), seitdem habe ich sie jedem ausgedehnteren Eingriff im Gebiet der Arterie vorausgeschickt. Es ist unverständlich, wenn gesagt wird, die Unterbindung des Gefäßes - zwischen A. thyreoida sup. und A. lingualis - erfülle nicht ihren Zweck. Natürlich operiert man auch so nicht wie bei Esmarchscher Blutleere. bei hyperämischen

1) Siehe Odontologisehe Blätter X, Nr. 3-4.

2) Fritz König, Zur Technik ausgedehnter Oberkieferresektionen. Langenb. Arch. f. klin. Chir., Bd. 61, H. 3. 
21) I. KöNıG, Über Prothesen bei Exartikulation u. Resektion d. Unterkiefers.

Individuen kommt immer noch Blut genug auf die Schnittfläche. Aber man braucht nur einmal, durch die Ausdehnung der Neubildung gezwungen, anf die andere Gesichtshälfte hinüberzukommen, um den Unterschied sofort zu bemerken.

Nun ist freilich die Arterie am genannten Punkt nicht immer so ganz leicht bloszulegen, man muß oft weit nach oben suchen. Gerade deshalb kann man sie aber gut von dem Schnitt aus erreichen, den wir zur Exartikulation machen. Man sieht zuweilen noch, daß die Narbe von der Unterlippenmitte abwärts zum Kinn, oder gar vom Mundwinkel nach abwärts geht, ehe sie in den Schnitt unter dem Kieferrand abbiegt, nach hinten führt sie zuweilen aufwärts vor dem Ohr bis zum Tragus. Das letztere kann durch die Ausdehnung der Geschwulst wohl einmal geboten sein, die ersteren beiden Schnittführungen sind unnötige Entstellungen. Wir beginnen am Kinn und führen bis vor den Proc. mastoideus einen nach abwärts stark bogenförmigen Schnitt, dessen Kuppe in Zungenbeinhöhe an der Stelle liegt, wo die A. lingualis unterbunden wird: von da aus finden wir die Carotis externa sicher. Der Lappen wird aufwärts präpariert und der Kiefer von unten her ausgehülst. Nach der Operation legt sich der Schnitt so weit abwärts, daß man, wie unsere Abbildung zeigt, außerordentlich wenig und beim Anblick von vorn überhaupt nichts von der Operation bemerkt. 